\title{
Germanica
}

\section{Das neue Europa und seine Nicht(s)-Orte}

Gekreuzte Perspektiven in Theatertexten von David Greig, Andrzej Stasiuk und Carles Batlle i Jordà

The new Europe and its nowhere places. Cross-perspectives in theatrical texts by David Greig, Andrzej Stasiuk and Carles Batlle I Jordà

La «nouvelle Europe » et ses lieux vacants. Perspectives croisées dans les textes de théâtre de David Greig, Andrzej Stasiuk et Carles Batlle I Jordà

\section{Elisabeth Tropper}

\section{OpenEdition}

Journals

Édition électronique

URL : http://journals.openedition.org/germanica/2906

DOI : 10.4000/germanica.2906

ISSN : 2107-0784

Éditeur

Université de Lille

Édition imprimée

Date de publication : 30 septembre 2015

Pagination : 95-110

ISBN : 9782913857353

ISSN : 0984-2632

Référence électronique

Elisabeth Tropper, "Das neue Europa und seine Nicht(s)-Orte », Germanica [Online], 56 | 2015, Online erschienen am: 30 September 2017, abgerufen am 06 Oktober 2020. URL : http://

journals.openedition.org/germanica/2906 ; DOI : https://doi.org/10.4000/germanica.2906

(c) Tous droits réservés 


\title{
Das neue Europa und seine Nicht(s)-Orte \\ Gekreuzte Perspektiven in Theatertexten von David Greig, Andrzej Stasiuk und Carles Batlle i Jordà
}

\author{
Elisabeth TROPPER \\ Université du Luxembourg, Universität Trier \\ „Ja, vielleicht wird mein künftiger EuroCity von \\ nirgendwo nach nirgendwo fahren." \\ (Andrzej Stasiuk, Logbuch)
}

\section{Einführende Bemerkungen}

\section{Das ,neue Europa'}

Wenn vom so genannten ,neuen Europa' die Rede ist, so wird in der Regel auf eine Vorstellung referiert, die sich bei näherer Betrachtung als höchst flexibel und bedeutungsoffen erweist - ,the imagination of an entity that might become, replace, or perhaps parasitically inhabit the

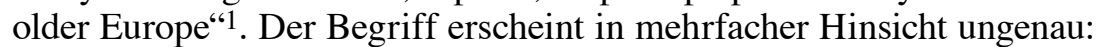
Nicht nur gestaltet sich das ,neue Europa je nach Perspektive immer wieder neu; schon der Begriff ,Europa selbst bezeichnet keineswegs

1. - Janelle G. Reinelt, „Performing Europe. Identity Formation for a ,New“ Europe“, in Theatre Journal 53/3 (2001), S. 365-387, S. 365. DOI: http://www.jstor. $\mathrm{org} / \mathrm{stable} / 25068949$ [5.4.2014]. 
eine klar definierte geographische oder politische Einheit, sondern vielmehr eine dynamische Vorstellung, einen „kollektive[n] imaginäre[n] Entwurf" 2 - oder, wie es im Rahmen neuerer transnational fokussierter Europa-Studien heißt: ,eine gedachte, raum-zeitliche Ordnung auf einer mittleren Ebene zwischen Nationalität und Globalität“"3.

Festzuhalten ist, dass die Vorstellung von einem ,neuen Europa mit der sukzessiven Transformation des europäischen Raumes und der ihn konstituierenden Nationalstaaten seit 1989 einhergeht, welche sich unter dem Stichwort ,Europäisierung (,Europeanization') als ,,a variety of political, social, economic and cultural processes that promote (or modify) a sustainable strengthening of intra-European connections and similarities through acts of emulation, exchange and entanglement" ${ }^{\text {"4 }}$ beschreiben lässt, wobei auch ,parallel processes of delimitation and ,othering", as well as fragmentation and conflict"5 eine zentrale Rolle spielen. Europäisierung meint demnach einen Prozess, der über den politisch-ökonomischen Zusammenschluss qua EU und Schengen-Raum hinausgeht, wobei sich das Unterfangen als janusköpfig erweist: Neben der Schwächung binneneuropäischer Grenzregimes sowie der nationalstaatlichen Einflusssphäre sind zugleich in ganz Europa Tendenzen einer (Re-)Nationalisierung und Partikularisierung zu verzeichnen, welche die Suche nach einer gemeinsamen europäischen Identität oder ,Seele, die den - überspitzt formuliert - heimatlos gewordenen nationalen Subjekten eine neue Identifikationsgrundlage bieten soll, immer dringlicher erscheinen lassen. Weitgehend ungeklärt bleibt, worin eine solche gemeinsame europäische Identität bestehen könnte; dass sie wohl allenfalls in der Vielfalt, den Dissonanzen und Brüchen zu finden sein dürfte ${ }^{6}$ als kollektiver, immer wieder aufs Neue zu verhandelnder und zu reflektierender Entwurf eben -, legt nicht zuletzt ein Blick auf die wechselhafte Geschichte des europäischen (Sub-)Kontinents nahe.

\section{Die theatrale Perspektive}

Wenn, wie die Herausgeber eines 2010 erschienenen Sammelbandes erklären, Europa „,seine Identität nicht zuletzt aus Ideen, Vorstellungen

2. - Hagen Schulze, Phoenix Europa. Die Moderne. Von 1740 bis heute, Berlin, Siedler (Siedler Geschichte Europas, Bd. 4), 1998, S. 513.

3. - Benjamin Drechsel et al., „Einleitung: Bilder von Europa aus kulturwissenschaftlicher Perspektive“, in Benjamin Drechsel et al. (Hrsg.), Bilder von Europa. Innenund Außenansichten von der Antike bis zur Gegenwart, Bielefeld, transcript (Europäische Horizonte 6), 2010, S. 11-35, S. 22.

4. - Ulrike von Hirschhausen, Klaus Kiran Patel, „Europeanization in History: An Introduction“, in Martin Conway et al. (Hrsg.): Europeanization in the Twentieth Century. Historical Approaches, Basingstoke, Palgrave Macmillan, 2010, S. 1-18, S. 2.

5. - Ebd., S. 3.

6. - Vgl. H. Schulze, a.a.O., S. 508. 
und Images [gewinnt], die sich unter dem Überbegriff des ,(Europa-) Bildes" subsumieren lassen"7, so arbeitet auch das Theater gestalterisch wie reflektierend an diesem Vorstellungsbild mit, indem es sich auf ästhetischer, struktureller wie institutioneller Ebene mit aktuellen europäischen Fragen und Problemstellungen auseinandersetzt, Europa-Diskurse bündelt und bricht, peripherisierte Perspektiven ins Zentrum rückt, Theatersprachen- und formen verflicht, Versuchsräume für alternative Konzepte eröffnet und/oder die grenzüberschreitende Zusammenarbeit sucht. Dass das Theater in besonderer Weise dazu geeignet ist, dem ,neuen Europa und seinen Implikationen künstlerisch $\mathrm{zu}$ begegnen, steht für die Theaterwissenschaftlerin Janelle Reinelt außer Frage: ,,[T]he issues confronting Europe involve matters of imagination, identification, tradition, and novelty: in short, suitable subjects for theatre's plastic skills." 8

Die im Folgenden untersuchten Theatertexte ${ }^{9}$ aus Schottland, Polen und Katalonien setzen beim Individuum an, das sich durch das ,neue Europa ${ }^{6}$ in einen akuten Krisenzustand versetzt sieht. In ähnlicher Weise, wie der von Hippokrates gestiftete Begriff ,krisis“ jene entscheidende Phase der Krankenbehandlung bezeichnet, die entweder zur Heilung des Patienten oder zu dessen Niedergang führt, entfalten sich die dramatischen Szenarien von David Greig, Andrzej Stasiuk und Carles Batlle i Jordà im Spannungsfeld zwischen Chance und Scheitern. Hinsichtlich ihrer Entstehungszeiten besteht eine Differenz von mehr als einer Dekade, wodurch sich der außerdramatische Referenzraum von der offiziellen Gründung der EU bis hin zu ihrer Osterweiterung erstreckt: David Greigs Europe wurde 1994 uraufgeführt, im Nachklang des Zerfalls der Sowjetunion und zwei Jahre nach der Unterzeichnung der Verträge von Maastricht, während Transit von Carles Batlle i Jordà und Warten auf den Türken von Andrzej Stasiuk in engerer zeitlicher Abfolge entstanden und uraufgeführt wurden, nämlich 2007 und 2009 - in einer Phase also, in der sich die Europäische Union gen Osten hin erweiterte und um eine gemeinsame Verfassung rang.

Die folgenden Überlegungen werden in erster Linie auf die textliche Ebene fokussieren, d.h. inhaltliche und formale Aspekte sowie intertextuelle Phänomene in den Vordergrund rücken; diese Einschränkung ist insofern erforderlich, als eine umfassendere, auch die Aufführung

7. - B. Drechsel et al., a.a.O., S. 11.

8. - J. G. Reinelt, a.a.O., S. 366.

9. - Wenn in einem umfassenden Sinne gemeint, wird im Folgenden dem Terminus Theatertext der Vorzug gegeben, um auch ,nicht mehr dramatische“ sowie ,postdramatische“ Zeichen miteinbezogen zu wissen (vgl. Gerda Poschmann, Der nicht mehr dramatische Theatertext. Aktuelle Bühnenstücke und ihre dramaturgische Analyse, Tübingen, Niemeyer, 1997 sowie Hans-Thies Lehmann, Postdramatisches Theater, Frankfurt am Main, Verlag der Autoren, 1999). 
miteinbeziehende Analyse den Rahmen des vorliegenden Beitrags sprengen würde. Das analytische Verfahren besteht hierbei in der gezielten Kreuzung mehrerer dramatischer bzw. theatraler Perspektiven auf das sich jeweils unterschiedlich konstituierende ,neue Europa', wodurch der Gegenstand nicht vereindeutigt, sondern vielmehr in seiner Ambiguität zwischen Nationalität und Transnationalität ebenso wie zwischen sozialpolitischer Realität und imaginärem Entwurf sichtbar gemacht werden soll.

\section{Theoretische Voraussetzung: Marc Augés Konzept der Nicht-Orte}

„Keine Analyse des sozialen Gefüges darf länger das Individuum verkennen, und keine Analyse des Individuums kann fortan die Räume ignorieren, durch die es sich hindurchbewegt"10, erklärt der französische Ethnologe Marc Augé in seinem 1992 erschienenen Werk Non-Lieux (Nicht-Orte). Dieses Individuum wiederum sieht Augé durch verschiedene „Figur[en] des Übermaßes“11 geprägt, darunter wesentlich auch durch ein „Übermaß an Raum"12, welches die Vermehrung von so genannten Nicht-Orten vorantreibe: Im Unterschied zum anthropologischen Ort ist der Nicht-Ort für Augé „ein Raum, der keine Identität besitzt und sich weder als relational noch als historisch bezeichnen lässt"13. Es handelt sich um Durchgangs- und Transiträume, zugehörig einer „Welt, die solcherart der einsamen Individualität, der Durchreise, dem Provisorischen und Ephemeren überantwortet ist" "14: Schnellstraßen, Autobahnkreuze, Flughäfen, Bahnhöfe, Shopping Malls, Durchgangslager. Sie kommunizieren ausschließlich über Texte und Anweisungen, die sich unterschiedslos an jeden von uns richten (,Wir wünschen Ihnen eine angenehme Reise") und die dadurch eine provisorische, mit den übrigen Passagieren oder Reisenden geteilte Identität erschaffen; einen, wie Augé ihn nennt, „Durchschnittsmenschen"15. Raum und Individuum lassen sich in den ephemeren und anbindungslosen Räumen des Transits voneinander losgelöst kaum denken; so nimmt es denn auch nicht wunder, dass das Theater gerade hier das desorientierte europäische Individuum par excellence gefunden $\mathrm{zu}$ haben scheint.

10. - Marc Augé, Nicht-Orte, Übersetzung von Michael Bischoff, 2. Aufl., München, Beck, 2011, S. 119f.

11. - Ebd., S. 47.

12. - Ebd., S. 39.

13. - Ebd., S. 83.

14. - Ebd.

15. - Ebd., S. 101. 


\section{Ein Theater der Nicht(s)-Orte}

\section{„We live in a dirty, nothing place“: David Greig, Europe (1994)}

Europe des schottischen Dramatikers David Greig, uraufgeführt 1994 am Traverse Theatre in Edinburgh, hat ein heruntergekommenes binneneuropäisches Grenzstädtchen zum Handlungsort - „A small decaying provincial town in Europe "16 Grenze keine Züge mehr halten, die Wirtschaft am Boden liegt und nebst Arbeitslosigkeit auch der Fremdenhass grassiert. An diesen Ort, der im Rahmen einer ausgeklügelten Geräuschdramaturgie immer wieder vom anschwellenden Lärm durchfahrender Züge geflutet wird, hat es zwei Flüchtlinge verschlagen: Sava und seine Tochter Katia, die aus einem nicht näher genannten Krieg in Europa geflohen sind und nun am Bahnhof der Grenzstadt festsitzen. Mit ihnen korrespondiert ein weiteres Vater-Tochter-Paar: der Bahnhofsvorsteher Fret, der nach wie vor seinen sinnlos gewordenen Dienst versieht, und seine Tochter Adele, die als Trainspotterin vom Bahnhofsdach aus sehnsüchtig die vorbeifahrenden Züge verfolgt. Als ,Daheimgebliebene“ stehen Fret und Adele den Exilierten Sava und Katia spiegelbildlich gegenüber.

Nach ersten Konflikten entwickelt sich zwischen den Männern eine Freundschaft, die vor allem auf der Entdeckung von Gemeinsamkeiten beruht: Beide sind ihrer Profession nach Mitarbeiter des staatlichen Bahnwesens und gehören einer Generation an, die ein sentimentales Verhältnis zu den industriellen Errungenschaften der Moderne pflegt; den neuen Zeiten (d.h. vor allem der zunehmenden Automatisierung von Arbeitsvorgängen) stehen sie skeptisch gegenüber. Katia wiederum wird zur Projektionsfläche für Adele, die in der ziellos Reisenden den erotisch aufgeladenen Gegenentwurf zu ihrer eigenen, als stumpf und perspektivlos empfundenen Lebenswirklichkeit zu erblicken vermeint: ,travel broadens the mind" 17 , ist sie überzeugt, und sie hat wenig Verständnis für die schmerzhafte Realität der Exilierten: „It doesn't broaden the mind, it stretches it like skin across a tannig rack... a pegged skin out to dry." 18

Die wachsende Arbeitslosigkeit ebenso wie die Sinnkrise, der die Bewohner angesichts der Bedeutungslosigkeit ihrer Stadt anheim gefallen sind (früher mussten sämtliche Züge zur Passkontrolle im Bahnhof halten, nun ist er zur reinen Durchfahrtsstation geworden), befeuern das xenophobe Klima, das sich zunehmend gegen die beiden Fremden richtet, die im funktionslos gewordenen Bahnhofsgebäude eine

16. - David Greig: Europe. In: Plays: 1, London, Bloomsbury, 2002, S. 1-90, S. 4.

17. - Ebd., S.53.

18. - Ebd. 
vorübergehende Bleibe gefunden haben. Um die Grenzstadt verlassen und ihre Reise unbehelligt fortsetzen zu können, beschafft Katia sich über den „Grenzverletzer"19 Morocco, einen weltläufigen Schmuggler, einen neuen Pass, während ihr Vater den Entschluss fällt zu bleiben: „I've found myself here. In a station. A station is a place to finish a journey as well [sic] a place to start one." 20

Katia und Adele verlassen zusammen die Stadt; ihre Väter bleiben zurück und fallen schließlich der aufgeheizten Stimmung zum Opfer: Die ehemaligen Fabrikarbeiter Horse und Berlin (Adeles Ehemann), die beispielhaft für die arbeitslos gewordene Arbeiterschaft der Grenzstadt stehen und deren zunehmende Radikalisierung im Handlungsverlauf des Stücks dramaturgisch mitvollzogen wird, werfen einen Brandsatz auf das Bahnhofsgebäude, das binnen kürzester Zeit in Flammen steht. Damit gelingt es ihnen, ihre im Verschwinden begriffene Stadt vorübergehend wieder sichtbar zu machen, sie gleichsam als Symbol in den öffentlichen Diskurs einzuschreiben: „They said the name of our town, politicians and sociologists all across the continent said its name. [...] Until it wasn't a name anymore but a condition, not a place but an effect. But it was our town." 21

\section{Vom Nicht-Ort zum Nichts-Ort}

Billy, der als Einziger der arbeitslos gewordenen Männer aufbricht, um sein Glück anderswo zu suchen, bezeichnet den Ort als „dirty, nothing place“22; und tatsächlich ist Greigs innereuropäische Grenzstadt vom Augé'schen Nicht-Ort des Transits zu einem Nichts-Ort, einem überflüssigen Flecken Erde geworden.

„Da Nicht-Orte durchquert werden, bemessen sie sich nach Zeiteinheiten. Keine Strecke ohne Fahrplan, ohne Anzeigetafeln für Ankunft und Abfahrt [...]. Man lebt dort ganz in der Gegenwart" ${ }^{\text {"23, }}$ konstatiert Marc Augé über die temporale Verfasstheit von Nicht-Orten. Diese „Herrschaft [...] der Aktualität“" ${ }^{4}$ hat in Europe bis zur Öffnung der Grenze nicht nur den Bahnhof, sondern die gesamte Gemeinschaft bestimmt. Seit der das städtische Leben strukturierende ,rhythm of the railway timetable“ 25 verstummt ist, scheint die Stadt mit einem Mal aller Zeitlichkeit enthoben; sie beginnt sich regelrecht aufzulösen, einer erodierenden Landschaft gleich: „It feels like things are crumbling

19. - Vgl. Eva Horn et al. (Hrsg.), Grenzverletzer. Von Schmugglern, Spionen und anderen subversiven Gestalten, Berlin, Kulturverlag Kadmos, 2002.

20. - D. Greig, a.a.O, S. 82.

21. - Ebd. S. 89.

22. - Ebd., S. 25.

23. - M. Augé, a.a.O., S. 104.

24. - Ebd.

25. - D. Greig, a.a.O., S. 6. 
[...] I've seen buildings go up and come down, I've seen street names change ... it's formed around me like geology. And now it's wearing away. It's eroding in the wind. Losing tiny particles of substance every day, getting smaller, breaking up.“26 Für Reisende ist die Grenzstadt nunmehr nichts weiter als eine $\mathrm{x}$-beliebige Durchfahrtsstation, deren vorbeifliegenden Namen man zur Kenntnis nimmt oder nicht; ihre Bedeutung als Knotenpunkt im Bahnstreckennetz, als Ort, an dem der Reisefluss unweigerlich unterbrochen werden muss, ist ihr im Zuge des europäischen Integrationsprozesses abhanden gekommen. Und nicht nur das: Das System der Strecken und Fahrpläne ist mit einem Mal selbst für Fret, den Eingeweihten, unverständlich geworden: „See this? Four hundred pages and none of it makes sense. Times, stations, trains... They've no relation to anything. Meaningless.“27 Das Eisenbahnnetz in Europe lässt sich folglich auch als ,an extended metaphor providing a tantalizingly problematic image of the European body politic" 28 verstehen.

Eines kollektiven Aktes der Neuerfindung bedürfend, zu dem die Bewohner sich jedoch außerstande sehen, verfällt die Stadt zusehends; selbst die Grenze zu der sie umgebenden Natur löst sich allmählich auf: Nachts dringen Wölfe aus den umliegenden Wäldern in die Stadt ein und marodieren durch die Straßen. Das ,neue Europa, das hier in Erscheinung tritt, ist der sich nach dem Zerfall der Sowjetunion in seinen Zugehörigkeiten neu formierende Subkontinent, dessen freier Binnenmarkt ebenso wie die kriegerischen Auseinandersetzungen am Balkan beträchtliche Migrationsströme freisetzen. Zugleich werden in Europe auch die Implikationen eines globalisierten Kapitalismus zum Thema gemacht: Zahlreiche Arbeitsplätze in der örtlichen Fabrik wurden gestrichen, um im internationalen Wettbewerb konkurrenzfähig zu bleiben.

Greig hat in seinen Text zwei gegenläufige Bewegungen eingeschrieben, anhand derer die Komplexität der Europäisierungsprozesse nach 1989 sichtbar wird: Den transnationalen Entwicklungen, im Rahmen derer die territorialen Binnengrenzen porös werden, steht die Sehnsucht nach klar definierten nationalen und regionalen Identitäten gegenüber, die als sinnstiftende Bezugspunkte in einer unübersichtlicher werdenden Welt herhalten können. Veränderung und Stasis, Mobilität und Immobilität, Destruktion und Affirmation sind die Pole, die sich in Greigs Krisenszenario nicht nur diametral zueinander verhalten, sondern zugleich überlagern und überformen. Für die Gemeinschaft

26. - Ebd., S. 77.

27. - Ebd., S. 11f.

28. - Clare Wallace, The Theatre of David Greig, London et al., Bloomsbury, 2014, S. 52. 
vor Ort stellen die migrierenden Figuren Sava und Katia nicht zuletzt deshalb eine Bedrohung dar, weil an ihnen diese Pole unvermeidlich zum Ausdruck kommen:

Dass die Einwanderer eine so starke (und oft so abstrakte) Furcht bei den Einheimischen auslösen, rührt vielleicht daher, dass sie ihnen zeigen, wie relativ die an den Boden geknüpften Gewissheiten sind. Eigentlich ist es der Auswanderer, der sie in der Person des Einwanderers beunruhigt und zugleich fasziniert. ${ }^{29}$

\section{„Wir sind die Waisen von Schengen“": Andrzej Stasiuk, Warten auf den Türken (2009)}

Der polnische Schriftsteller Andrzej Stasiuk zeichnet in seinem Theatertext Czekajac na Turka (Warten auf den Türken, uraufgeführt 2009 am Stary Teatr in Krakau, in deutscher Übersetzung von Olaf Kühl als Stückabdruck in Theater Heute erschienen) in ähnlicher Weise die Orientierungslosigkeit einer Gemeinschaft nach, deren Lebensinhalt durch die Existenz einer bewachten Grenze gewährleistet war - symbolisiert durch einen rot-weiß-roten Schlagbaum. Das Motiv ist vertraut: Im massenmedialen Diskurs stellt der sich öffnende Grenzbalken ein beliebtes Bildmotiv für den Wegfall der Grenzen zwischen EU-Mitgliedstaaten dar ${ }^{30}$. Seit die Grenze offen ist (hier ganz konkret die polnisch-slowakische Grenze, an der seit der Schengen-Erweiterung von 2007 keine Pass- und Zollkontrollen mehr durchgeführt werden ${ }^{31}$ ), haben Stasiuks Figuren ihre Aufgabe verloren. Dennoch verharren sie an diesem bedeutungslos gewordenen Ort: der Grenzwächter Edek (eine an den Bahnhofswärter Fret gemahnende Figur) ebenso wie die Kioskbesitzerin Marika und der Chor der alten Schmuggler. Gemeinschaftlich betrauern sie den Verlust ,ihrer' Grenze:

Wir sind die Waisen von Schengen. Wir sind ohne Leben. Man hört unsere Stimmen, jedoch Taten vollbringen wir nicht, denn es gibt keine Grenzen mehr. Wir sind ohne Leben, man hört nur unseren Gesang, unser Schengener Klagelied, unseren Waisenchor. ${ }^{32}$

Zwei junge Mitarbeiter einer privaten Wachschutzfirma erscheinen, um die inzwischen in Privatbesitz übergegangene Grenzregion zu bewachen; wie sich herausstellt, hat eine türkische Geschäftsfrau

29. - M. Augé, a.a.O., S. 118f.

30. - Vgl. Petra Mayrhofer, ,,Festung Europa“? Grenzikonografien im europäischen Raum“, in B. Drechsel et al., a.a.O., S. 307-319, S. 313.

31. - Vgl. Barbara Burkhardt, „Marika links, Marika rechts“, in Theater Heute 8/9 (2009), S. 52-55, S. 53.

32. - Andrzej Stasiuk, Warten auf den Türken, Übersetzung von Olaf Kühl, Stückabdruck, in Theater Heute 8/9 (2009), S. 10. 
das Gebiet zu beiden Seiten der Grenze aufgekauft, um daraus einen Themenpark unter dem Motto „Grenzübergang im alten Osteuropa“33 zu machen. Als der kleinen, hinfällig gewordenen Grenzgemeinschaft angeboten wird, Teil des Re-Enactments mit „Stacheldraht, Hunde[n], Kontrollen, Schusswechsel, Folter und so weiter" ${ }^{\text {"34 }} \mathrm{zu}$ werden, geben die Verbliebenen ihre Stellung auf und ziehen fort. Wohin, erfahren wir nicht.

\section{Identität und Grenze}

Im Zentrum von Stasiuks dramatischer Versuchsanordnung steht „die aporetische Struktur jeder Grenze als Bedingung von Identität und Heimat sowie von Übertretung" 35 . Territoriale Grenzen werden wesentlich durch ihre Überschreitung markiert und stabilisiert, und diese Überschreitung ist - bzw. war, wie im Hinblick auf den Schengenraum gesagt werden muss - an bestimmte performative Akte geknüpft. In Warten auf den Türken finden diese Akte Ausdruck im Symbol des Schlagbaums, dessen Heben und Senken bis zur Öffnung der Grenze die Weiterfahrt regulierte: ,Ein Schlagbaum, das ist Leben! Geordnete Bewegung. Nach oben heißt - freie Fahrt. Nach unten - absoluter Halt!"36

Zwar wurden, wie die Herausgeber eines Sammelbandes über Grenzen in Europa zu Recht feststellen, durch das Schengener Abkommen die binneneuropäischen Grenzkontrollen, nicht jedoch die Grenzen selbst abgeschafft ${ }^{37}$; wenn Grenzen jedoch letztlich ,,nur in actu, als technische Vorrichtungen und soziale Arrangements des Aus- und Einschließens wie des Öffnens" 38 existieren, so stellt sich dennoch die berechtigte Frage, ob mit der Suspendierung der an den Grenzübertritt gekoppelten performativen Akte nicht ein Bedeutungsverlust einhergeht, der einer Abschaffung der Grenze zumindest nahekommt.

Eine besondere Bedeutung besitzt die Grenze für das sich spiegelbildlich entsprechende Paar Grenzschützer-Grenzverletzer. Da „Konformität und Abweichung, geregelter Grenzverkehr und Grenzvergehen [...]

33. - Ebd.

34. - Ebd.

35. - Franziska Schößler, „Mental Map des Globalen. Theatrale Grenzverhandlungen und Prekarität bei Christoph Schlingensief und Andrzej Stasiuk", in Natalie Bloch et al. (Hrsg.), Theater International. Eine Vortragsreihe, Luxemburg, Hydre Éditions, 2014, S. 19-44, S. 35.

36. - A. Stasiuk, a.a.O., S. 2.

37. - Vgl. Michael Gehler, Andreas Pudlat, „Vorwort“, in Michael Gehler et al., Grenzen in Europa, Hildesheim et al., Olms, 2009, S. 7-14, S. 7.

38. - Stefan Kaufmann, Ulrich Bröckling, Eva Horn, „Einleitung“, in Eva Horn et al., a.a.O., S. 7-22, S. 7. 
wechselseitig ineinander eingeschrieben“39 sind, haben mit der Öffnung der Grenze sowohl Edek (als Grenzschützer) als auch der Chor der Schmuggler (als Grenzverletzer) ihre Funktion verloren. Den Schmugglern war die Grenze bis dato vor allem als ökonomische Barriere von Nutzen, da sie von den Zöllen und Warenverboten profitierten, die auf der jeweils anderen Seite Geltung besaßen. Nun droht der Ort in ähnlicher Weise zum Nichts-Ort zu werden, wie Greig dies anhand der kleinen Grenzstadt in Europe vorgeführt hat. Während deren Bewohner dem Auflösungsprozess vorübergehend Einhalt gebieten, indem sie sich durch einen fremdenfeindlichen Gewaltakt in das mediale Gedächtnis der Gegenwart einschreiben, steht am Ende von Warten auf den Türken das rückwärtsgewandte Vorhaben, einen vergangenen Zustand zu rekonstruieren und zum Unterhaltungszweck einzufrieren. Bei diesem Akt der Musealisierung geht es jedoch weniger um historische Authentizität denn um spezifische Vorstellungen und Phantasmagorien vom ,alten Osteuropa'. Edeks Versuch, die falschen Auffassungen zu berichtigen („Hier war kein Stacheldraht. Den gab es an der Russengrenze.“40), findet bei der Geschäftsfrau kein Gehör.

Ähnlich wie Europe, führt auch Warten auf den Türken die paradoxe Gleichzeitigkeit von Transnationalisierungs- und Renationalisierungsprozessen vor, wobei in Stasiuks innereuropäischer Bestandsaufnahme zuweilen eine leise Sehnsucht nach der verlorenen Grenze anklingt. Der die ansonsten unsichtbare Trennlinie markierende Schlagbaum besitzt für den alten Grenzer Edek eine geradezu magische Funktion; doch wie Fret, der sich im komplexer gewordenen System der Fahrpläne nicht mehr zurechtfindet, hat Edek die Kontrolle über ,seinen' Grenzbalken verloren: In der Krakauer Uraufführung durch Mikołaj Grabowski arbeitet sich der Schauspieler Jan Peszek regelrecht körperlich an ihm ab, ohne etwas gegen sein nun unreguliertes Heben und Senken ausrichten zu können.

\section{„I was born under a wand'rin' star“: Carles Batlle i Jordà, Transit (2007)}

In seinem Theatertext Transit (uraufgeführt 2007 in der Sala Beckett in Barcelona) überschreibt der katalanische Autor und Dramaturg Carles Batlle i Jordà die Situation Kataloniens mit jener der Europäischen Union und deren Selbstfindungs- und Migrationsbewegungen. Diese doppelte Perspektive erklärt Batlle als konstitutiv auch für die eigene Biographie: ,Ich fühle mich [...], wenn ich von meiner unsicheren katalanischen Identität aus schreibe, voll und ganz als Europäer."41

39. - Ebd., S. 8.

40. - A. Stasiuk, a.a.O., S. 10.

41. - „Carles Batlle im Gespräch“, in Carles Batlle i Jordà, Transit, Übersetzung 
Ort der Handlung von Transit ist das Abteil eines Fernzuges, der Richtung Norden fährt; die Zeit: eine „nicht sehr ferne Zukunft“42. Mehrere Personen mit krisenhaften Identitäten treffen hier aufeinander: der Schaffner Tort, der in Rente geschickt werden soll und sich neben Fret und Edek in die Reihe obsolet gewordener Vertreter einer alten Ordnung einreiht; ein herzkranker Mann, der seine Herkunft nicht kennt, und seine Tochter, die von ihm schwanger ist; ein Junge, der als blinder Passagier reist; und nicht zuletzt eine Frau mit traumatischen Erinnerungen und einer Bombe im Gepäck.

Die Szenen des handlungsarmen Stücks sind in einander verschachtelt und folgen einer ruckhaften Dramaturgie aus Zeitsprüngen, Reprisen und Variationen. Am Ende schaffen lediglich Nina (die junge Frau) und Pol (der blinde Passagier) den Absprung aus dem Zug und somit in eine alternative Zukunft; die lange antizipierte Bombe reißt die übrigen Passagiere in den Tod: „Plötzlich grelles Licht" ${ }^{\text {"43 }}$, lautet die letzte Regieanweisung. Mit der Explosion fallen das Ende der dramatischen Handlung und das Ende des Theaterereignisses zusammen; die vorübergehend zustande gekommene Reisegemeinschaft ist vernichtet, und nur ihre jüngsten Mitglieder dürfen weiterleben. Hierin stimmen die Entwürfe von Batlle i Jordà und Greig in erstaunlicher Weise überein: Die Jungen erhalten auf dem Höhepunkt der Krise die Chance, ihr Leben radikal neu zu entwerfen, während die Alten nicht nur ad acta gelegt, sondern regelrecht ausgelöscht werden. (Ganz anders wiederum bei Stasiuk, der die alte Generation weiterziehen lässt, während der junge Wachschutzbedienstete Patryk im Gerangel versehentlich von seiner Kollegin erschossen wird.)

\section{Identität und Kultur}

Batlle parallelisiert die Reisebewegung seiner Figuren mit einer diffusen Sehnsucht nach Identität und Heimat - oder vielmehr nach dem, was sie dafür halten. Marius ist mit seiner Tochter auf dem Weg zurück zu seinen vermeintlichen Wurzeln; zu einem Ort, den er nicht kennt, in eine Sprachwelt, die er bislang vor ihr verschlossen gehalten hat - weil er, wie er am Ende bekennt, selbst keinen Zugang mehr zu ihr hat. Während er nichtsdestotrotz an der Hoffnung festhält, am Ende seiner Reise auf ein Moment der Vertrautheit und des Wiedererkennens zu stoßen, glaubt seine Tochter nur an den steten Wandel, der alle Wurzeln ausreißt. Der ,panoramatische Blick“44 aus dem Zugfenster,

von Thomas Sauerteig und Hans Richter, Gifkendorf, Merlin, 2007, S. 7-16, S. 7.

42. - Carles Batlle i Jordà, Transit, in C. Batlle i Jordà, a.a.O., S. 17-108, S. 27 f.

43. - Ebd., S.108.

44. - Wolfgang Schivelbusch, Geschichte der Eisenbahnreise. Zur Industrialisierung von Raum und Zeit im 19. Jahrhundert, 5. Aufl., Frankfurt am Main, 
der die Landschaft in Gestalt einer sich kontinuierlich transformierenden „Bilder- und Szenenfolge“ 45 vorbeifliegen lässt, entspricht dem flexiblen und anbindungslosen Lebenskonzept, das die junge Frau sich angeeignet hat:

Schau raus, schau raus ... schau aus dem Fenster ... Okay? Gut, was siehst du? Bäume? Bäume, sehr gut ... die Bäume, die ganze Landschaft ... das alles verschwindet. Schau, ist nicht mehr da ... ist nicht mehr da ... ist nicht mehr da ... [...] Alles was du gesehen hast, ist jetzt schon Vergangenheit [...] das alles schläft nicht ein und wartet, bis der verlorene Sohn sich irgendwann entscheidet zurückzukommen. Das alles verändert sich. ${ }^{46}$

Es ist eine transitäre Landschaft, eine Landschaft des Übergangs, die mit dem Zustand der sie betrachtenden Figuren korrespondiert. Auch bei Batlle i Jordà besteht eine enge Verbindung zwischen dem Individuum und den es umgebenen Räumen; sämtliche Bereiche des Textes durchdringt ein Eindruck von Lückenhaftigkeit und Dislozierung, der sich bis in den formalen Aufbau mit seinen Pausen und Brüchen fortsetzt.

Angesichts einer sich wandelnden europäischen Landschaft mit ihren fluktuierenden Grenzen und Migrationsbewegungen sieht die Romanistin Sharon G. Feldman in Batlles Theatertexten eine ,transkulturelle Sehnsucht am Werke, ,upon which there is engraved an aspiration to transcend the local and the particular"47. Das Spannungsverhältnis zwischen Globalisierungs- und Internationalisierungstendenzen auf der einen sowie regionalen Abspaltungs- und Partikularisierungsbestrebungen auf der anderen Seite bleibt in Transit jedoch ungelöst; die Mobilität und Ungebundenheit der Figuren erscheint durchweg als Problem. Der Autor bestärkt diesen Eindruck, wenn er in einem Interview, das der in Buchform publizierten deutschen Übersetzung des Theatertextes vorangestellt ist, erklärt: „Die katalanische Kultur befindet sich in einer prekären Situation, sie ereignet sich in einer Welt, die geprägt ist von Vermischung.“" ${ }^{8}$ Die Katalanen seien, so führt er weiter aus, „hin und her gerissen zwischen der Faszination für das Andersartige, dem drängenden Wunsch nach Vermischung einerseits, und der Treue zur Tradition, zur Sprache, zu all dem, was Identität stiftet, andererseits“. 49 Skizziert wird hier ein Konzept von Identität, das eine klare Abgrenzung

$\overline{\text { Fischer, 2011, S. } 60 .}$

45. - Ebd.

46. - C. Batlle i Jordà, a.a.O., S. 27f.

47. - Vgl. Sharon G. Feldman, In the Eye of the Storm. Contemporary Theatre in Barcelona, Lewisburg, Bucknell University Press, 2009, S. 257f.

48. - C. Batlle i Jordà, a.a.O., S. 7.

49. - Ebd. 
voraussetzt, welche sich jeder Öffnung oder ,Vermischung' enthält; wo diese stattfindet, versetzt sie - so ließe sich der Gedanke fortsetzen, und so legt es auch der untersuchte Theatertext auf den ersten Blick nahe das bis dato mit sich identische Individuum in die Krise. Gleichzeitig demontiert Batlle in Transit solche geschlossenen Identitätskonzepte ebenso wie essentialistische Konzepte von Kultur, mit denen zweifelhafte Vorstellungen von Reinheit und Unvermischtheit einhergehen, und führt sie als trügerisch vor: Nicht zuletzt anhand der dysfunktionalen inzestuösen Beziehung zwischen Vater und Tochter wird deutlich, dass eine Ordnung, die auf dem Ausschluss all dessen, was als ,fremd angesehen wird, aufbaut, zwangsläufig zum Scheitern verurteilt ist. Die Durchlässigkeit und Uneindeutigkeit von Identitätskonzepten mag in Transit krisenhafte Zustände hervorrufen; diese erscheinen jedoch angesichts eines sich wandelnden europäischen Raums sowohl unausweichlich als auch unerlässlich.

\section{Gekreuzte Perspektiven: Schnittpunkte und Abweichungen}

Wie eingangs erläutert, bildet die Vorstellung von einem ,neuen

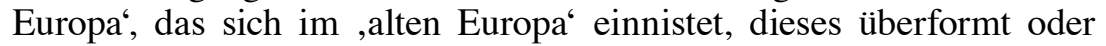
sich einverleibt, eine bedeutungsoffene und wandelbare Konstruktion, deren spezifische Gestalt neben überregionalen bzw. transnationalen Tendenzen und Wechselwirkungen zugleich an bestimmte historische und topographische Kontexte gebunden ist. Unter lokal-spezifischen Gesichtspunkten betrachtet, entwerfen David Greig und Carles Batlle i Jordà ihre dramatischen Szenarien im Spannungsfeld der (durch die europäische Integration nicht minder gewordenen) Unabhängigkeitsbestrebungen ihrer Heimatregionen Schottland und Katalonien, während Andrzej Stasiuk vom erklärten Standpunkt eines „mittelosteuropäische[n] Provinzler[s]" 50 schreibt, der sich, wie der Autor in einem Interview erläutert, mit einer anderen Perspektive ausgestattet sieht ,als ein typischer Vertreter des Westens" 51 und den europäischen Subkontinent in zwei Teile gespalten sieht: „Ich denke, dass diese ,zwei Europas، aus einem gewissen Abstand gegenseitig ihren langsamen, sanften Untergang betrachten werden. Das eine wird an Übersättigung, das andere an einem Mangel an Europa sterben." 52 Der geplante Vergnügungspark in Warten auf den Türken zementiert die

50. - Bernhard Hartmann, ,Eine lange, schmerzlose Agonie. Andrzej Stasiuk über den sanften Untergang der ,zwei Europas ““, in Theater der Zeit 11 (2006), S. 53.

51. - Ebd.

52. - Ebd. 
imaginäre Bruchlinie zwischen diesen beiden Europas, indem er ,den Osten' als das innereuropäische Andere stilisiert und einfriert.

Setzt man die bereits in den Einzelanalysen begonnene Verflechtung der Texte fort und isoliert hierbei einzelne Punkte der Überschneidung und Abweichung, so ist zunächst festzuhalten, dass es sich in formaler Hinsicht um drei höchst unterschiedliche Arbeiten handelt: Warten auf den Türken ist ein weitgehend konventioneller Dramentext, während Europe mit Brecht'scher Verfremdung ebenso operiert wie mit herkömmlichen Spielszenen und Momenten einer geradezu lyrischen Bildhaftigkeit; Transit wiederum zeichnet sich, wie bereits skizziert, durch eine (größtenteils postdramatisch zu nennende) Dramaturgie der Leerstellen, Zeitsprünge und Wiederholungen aus.

Hinsichtlich der Ähnlichkeiten und Überschneidungen fällt zuallererst die Figurenkonstellation ins Auge, die so etwas wie einen kollektiven Angelpunkt bildet: Alle drei Autoren setzen eine ältere Männerfigur ein, in der die alte und inzwischen überkommene Ordnung ihre Verkörperung findet; eine Figur, deren Hauptaufgabe in der Kontrolle (der Grenze, der Pässe, der Fahrpläne, der Zugtickets) besteht und die eine an Fetischismus grenzende Beziehung zu den mit dieser Kontrollfunktion verbundenen Objekten (Gleisanlange, Schlagbaum, Schaffnerzange) aufweist. Und als gehörten sie demselben dramatischen Universum an - oder als ließe sich ein kurzer Name leichter wegwischen aus den Chroniken der Gegenwart -, heißen sie Fret, Edek und Tort.

Daneben nimmt die Generationenfrage eine zentrale Rolle in der Figurenkonstellation ein: sei es über Vater-Tochter-Paare wie bei Greig und Batlle i Jordà, sei es in Form einer symbolischen Vater-SohnBeziehung wie im Falle Edeks und seines jungen Nachfolgers Patryk in Warten auf den Türken. Der in den Generationenkonflikt weitergetragene europäische Wandel nimmt jedoch, wie bereits erwähnt, bei Stasiuk eine von den anderen beiden Szenarien abweichende Wendung, indem er die hinfällig gewordenen Vertreter der alten Ordnung überleben und in eine - wiewohl ungewisse - Zukunft aufbrechen lässt.

Eine auffällige Übereinstimmung zwischen den Texten von Greig und Stasiuk wiederum besteht im Einsatz von Chorfiguren: In Europe setzt sich dieser Chor aus Bürgern der Grenzstadt zusammen, in Warten auf den Türken aus einer Gruppe alter Schmuggler. Gemäß den Chorfiguren des späteren antiken Dramas beobachten und kommentieren auch die Chöre bei Greig und Stasiuk das Geschehen, ohne je in die Handlung einzugreifen. In Warten auf den Türken thematisiert der Chor der alten Schmuggler (dessen hoher Ton durchaus ironische Anklänge aufweist ${ }^{53}$ )

53. - Vgl. F. Schößler, a.a.O., S. 35. 
seine auf Kommentar und Interpretation beschränkte dramaturgische Funktion sogar selbst: „Ehrlich gesagt, kannst du uns mal, wir sind der Chor und nehmen nicht an der Handlung teil. Du darfst uns nur zuhören, also pass auf [...].“54

Der Theaterwissenschaftlerin Ulrike $\mathrm{Haß}$ zufolge ist der antike Chor geprägt durch die „Figur des ,Schon-da““55, welche zunächst ,,mit einer ,Wo'-Frage zusammenhängt, die den ,Ort ${ }^{*}$ als Befragten fremd macht und zu einem Gegenstand der Reflexion werden lässt“"56. Des Weiteren sei dieser Figur „eine bestimmte zeitliche Struktur eigen, die Zeitlichkeit der Vorgängigkeit und Dauer, die sich weniger zur Gegenwart entschließt, als dass sie eine in gewisser Weise , anfangs-

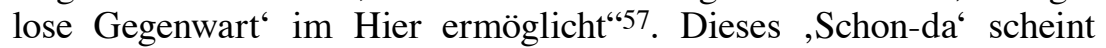
den zeitgenössischen Chören von Greig und Stasiuk zusehends zum Nachteil zu gereichen; zugehörig einem Raum, dessen Bedingungen einem fundamentalen Wandel unterworfen sind, werden sie - wie auch die Protagonisten - von den Veränderungen überrollt und in ihrem Selbstverständnis erschüttert. Es zeigt sich, dass Raum und Individuum nicht unabhängig voneinander zu denken sind; ihre Krisenzustände beeinflussen und bedingen einander. Folglich korrespondieren in allen drei Theatertexten Momente der Transgression territorialer Grenzen mit einer grundsätzlichen Durchlässigkeit von Identität, ob diese von den Figuren gewollt sein mag oder nicht.

Nicht zuletzt, und dies stellt eine wesentliche Voraussetzung für den vorliegenden Beitrag dar, manifestiert sich das ,neue Europa' bei Greig, Stasiuk und Batlle i Jordà in auffallender Weise an Nicht-Orten des Transits bzw. an Grenzübergängen, die - ihrer spezifischen Funktion als Nadelöhre des regulierten Grenzübertritts verlustig gegangen zu bedeutungslosen Nichts-Orten geworden sind. Hierbei stellen die Handlungsorte von Europe und Transit Nicht-Orte im Sinne Marc Augés par excellence dar: In Europe ist es der Bahnhof als klassischer Verkehrs- bzw. Transitraum, in Transit das Abteil eines Fernzuges jener „Raum des Reisenden“ also, der für Augé den „Archetypus des Nicht-Ortes" ${ }^{\text {"58 }}$ darstellt.

\section{Schluss}

Die für den vorliegenden Beitrag untersuchten Theatertexte von David Greig, Andrzej Stasiuk und Carles Batlle i Jordà setzen

54. - A. Stasiuk, a.a.O., S. 8.

55. - Ulrike Haß, „Woher kommt der Chor“, in Maske und Kothurn 3 (2012), S. 13-30, S. 19.

56. - Ebd., S. 26.

57. - Ebd.

58. - M. Augé, a.a.O., S. 90. 
bei der Herausforderung an, politische, nationale und kulturelle Identität im Kontext der europäischen Integration neu zu denken, und brechen die Implikationen sich wandelnder Raumsemantiken auf Figurenkonstellationen und dramatische Konflikte herunter. Über die versuchte Überlagerung (oder Kreuzung) der dramatischen Perspektiven tritt nicht nur ein vielschichtiges ästhetisches Wissen über das ,neue Europa' mit seinen veränderten raumsemantischen Bedingungen zutage; darüber hinaus wird eben jenes komplexe und zuweilen dissonante Konstrukt manifest, das Europa als ,kollektiven imaginären Entwurf ${ }^{‘}$ im Spannungsfeld zwischen Nationalität und Globalität ausmacht. In dieser Hinsicht lösen die Texte ein, was Patrice Pavis als unerlässlich für eine interkulturelle Theaterpraxis auf der Höhe der Zeit erklärt hat: „We now have to conceptualize national or cultural belonging differently, we have to reveal its inconsistency, its myth, its mystification."59

59. - Patrice Pavis, „Intercultural Theatre today“, in Forum Modernes Theater 25/1 (2010), S. 5-15, S. 14. 